\title{
Atenção domiciliar: Desafios para a promoção da saúde de idosos
}

\author{
Home care: Challenges for the promotion of elderly health \\ Atención en el hogar: Desafíos para la promoción de la salud de los ancianos
}

Recebido: 10/03/2021 | Revisado: 17/03/2021 | Aceito: 20/03/2021 | Publicado: 27/03/2021

\author{
Késia Larissa Brito Coutinho \\ ORCID: https://orcid.org/0000-0003-3520-708X \\ Universidade Federal do Pará, Brasil \\ E-mail: kesia.coutinho@altamira.ufpa.br \\ Francisco Bruno Teixeira \\ ORCID: https://orcid.org/0000-0001-8596-3727 \\ Universidade Federal do Pará, Brasil \\ E-mail: Teixeira.f.bruno@gmail.com
}

\begin{abstract}
Resumo
O presente artigo tem por objetivo identificar a importância da Atenção Domiciliar (AD), juntamente com os desafios dessa modalidade terapêutica na promoção da saúde de pessoas idosas. A metodologia constituiu-se de uma revisão de literatura na modalidade narrativa, que apresenta um caráter descritivo-discursivo. Utilizou-se como fonte de pesquisa as bases de dados BVS, SciELO e LILACS. A ocorrência de atendimento domiciliar é maior em mulheres, idosas, com doenças crônicas (como hipertensão, diabetes) associadas a comorbidades e histórico de quedas e hospitalização. Os resultados apontam para uma necessidade de mudança do modelo institucionalizado de saúde, redução dos custos hospitalares e uma necessidade do cuidado centrado no paciente e em sua relação com o próprio espaço de convivência através da AD. Concomitante a isso, resultados consignam a necessidade de enfrentamento dos desafios que ela traz como o desgaste incessante do cuidador, o qual tem poucas informações e orientação de como cuidar, além das próprias dificuldades e barreiras do território do idoso, difícultando o cuidado e tornando o quadro epidemiológico mais complexo. Conclui-se a necessidade de aprofundamento de discussão de várias questões que abrangem o AD, e a importância de reestruturação do acesso com ênfase na integralidade da saúde do idoso e nos atores importantes nesse processo: o cuidador, a família e a equipe de saúde.
\end{abstract}

Palavras-chave: Idosos; Serviços de assistência domiciliar; Serviços de saúde para idosos; Atenção primária à saúde.

\begin{abstract}
This article aims to identify the importance of Home Care (HC), together with the challenges of this therapeutic modality in promoting the health of the elderly. The methodology consisted of a literature review in the narrative modality, which has a descriptive-discursive character. The BVS, SciELO and LILACS databases were used as a research source. The occurrence of home care is higher in women, elderly women, with chronic diseases (such as hypertension, diabetes) associated with comorbidities and a history of falls and hospitalization. The results point to a need to change the institutionalized health model, to reduce hospital costs and a need for patient-centered care and its relationship with the living space itself through HC. Concomitant to this, results consign the need to face the challenges that it brings such as the incessant weariness of the caregiver, who has little information and guidance on how to care, besides the own difficulties and barriers of the elderly's territory, making care difficult and making more complex epidemiological picture. It concludes the need to deepen the discussion of several issues that cover HC, and the importance of restructuring access with an emphasis on the integrality of the elderly's health and the important actors in this process: the caregiver, the family and the health team.
\end{abstract}

Keywords: Elderly; Home care services; Health services for the elderly; Primary health care.

\section{Resumen}

Este artículo tiene como objetivo identificar la importancia de la Atención Domiciliaria (HC), junto con los desafíos de esta modalidad terapéutica en la promoción de la salud de las personas mayores. La metodología consistió en una revisión de la literatura en la modalidad narrativa, que tiene un carácter descriptivo-discursivo. Se utilizaron las bases de datos de BVS, SciELO y LILACS como fuente de investigación. La ocurrencia de cuidados domiciliarios es mayor en mujeres, ancianas, con enfermedades crónicas (como hipertensión, diabetes) asociadas a comorbilidades y antecedentes de caídas y hospitalización. Los resultados apuntan a la necesidad de cambiar el modelo de salud institucionalizado, para reducir los costos hospitalarios y la necesidad de una atención centrada en el paciente y su relación con el propio espacio vital a través de la HC. Concomitante a esto, los resultados consignan la necesidad de afrontar los retos que conlleva como el cansancio incesante del cuidador, que tiene poca información y orientación sobre cómo cuidar, además de las dificultades y barreras del territorio del anciano, dificultando el cuidado. y haciendo más difícil un cuadro epidemiológico más complejo. Se concluye la necesidad de profundizar en la discusión de 
varios temas que cubren la EA, y la importancia de reestructurar el acceso con énfasis en la integralidad de la salud de las personas mayores y los actores importantes en este proceso: el cuidador, la familia y el equipo de salud.

Palabras clave: Ancianos; Servicios de atención domiciliaria; Servicios de salud para ancianos; Atención primaria de salud.

\section{Introdução}

O aumento da longevidade e da expectativa de vida é uma realidade cada vez mais frequente na população Brasileira. De acordo com o Ministério da Saúde, para o ano de 2050, a expectativa no Brasil é de que existirão mais idosos que crianças abaixo de 15 anos. Porém, o aumento do envelhecimento populacional interfere em suas condições de saúde, podendo elevar as enfermidades crônicas com comorbidades, intensificando, consequentemente, a demanda por assistência especializada e de alto custo (Miranda, et al., 2016; Guimarães, 2008).

O mundo todo está envelhecendo. Este é um fato que as nações estão começando a compreender à medida que buscam caminhos para manter seus cidadãos idosos socialmente e economicamente integrados e independentes. No entanto, os sistemas nacionais de saúde enfrentam dificuldades para tornar estes novos tratamentos acessíveis à maioria das pessoas (Kalache, 2008). Precisa-se reorganizar os níveis de cuidado para atender às necessidades, além de inovar e tomar por base experiências de outros países que já vivenciaram o processo de envelhecimento (Miranda, et al., 2016).

Uma das alternativas para o sistema de saúde é a adesão do cuidado domiciliar, inserida dentre as políticas públicas, pois as incapacidades e fragilidades dos idosos exigem ações integrais do sistema de saúde como preconiza a Assistência Domiciliar. Ela está presente na formulação das políticas públicas de saúde e de assistência social, na gestão e nas demais práticas em serviços de saúde (Silva, et al., 2010; Miranda, et al., 2016).

A Atenção Domiciliar, a qual se insere dentro da Assistência Domiciliar, é um conjunto de ações realizadas por uma equipe interdisciplinar no domicílio do usuário, a fim de restabelecer sua independência e a preservação de sua autonomia, além da possibilidade de desospitalização, com liberação de leitos para doentes que realmente deles necessitam, redução de complicações causadas pela hospitalização de longo prazo e redução dos custos de todo o processo de hospitalização (Brasil, 2006), visto que temos um heterogêneo quadro epidemiológico do brasil, prevalecendo as doenças crônicas não transmissíveis (DCNT). A atenção domiciliar teria o potencial de ampliar o acesso aos serviços dos idosos com incapacidades, humanizar o cuidado e fortalecer o vínculo das equipes de saúde com a população (Wachs, et al., 2016).

A Resolução RDC no 11/2006 da Agência Nacional de Vigilância Sanitária (Anvisa)/Ministério da Saúde (MS) aprovou o Regulamento Técnico para o funcionamento dos Serviços de Atenção Domiciliar no Brasil, envolvendo ações de promoção à saúde, prevenção, tratamento de doenças e reabilitação desenvolvidas em domicílio.

Essa modalidade terapêutica vem avançando desde sua implementação. Porém, com o passar do tempo, surgem situações conflituosas e desafios entre os diversos atores envolvidos no contexto da atenção domiciliar. Como a sobrecarga de trabalhos do cuidador domiciliar em sua condição de exercício contínuo de tarefas (Guerra, et al., 2017), principalmente, segundo Carvalho, et al., (2001) quando os cuidadores são familiares que, despreparados, acabam também necessitando de cuidados. Além do espaço no atendimento em domicílio o qual pode ser restrito, exigindo aumento de despesas familiares.

Nesse sentido, este artigo objetiva identificar a importância da Assistência domiciliar, juntamente com os desafios dessa modalidade terapêutica na promoção da saúde de pessoas idosas.

\section{Metodologia}

Trata-se de um estudo de revisão de literatura na modalidade revisão narrativa, que apresenta um caráter descritivodiscursivo, caracterizando-se pela ampla apresentação e discussão de temas de interesse científico, constituindo-se num importante elemento na literatura científica (Munoz, et al. 2002). 
Utilizou-se como fonte de pesquisa as bases de dados Biblioteca Virtual em Saúde (BVS), Scientific Electronic Library Online (SciELO), Literatura Latino-Americana e do Caribe em Ciências da Saúde (LILACS), encontradas produções bibliográficas de idioma nacional e internacional nos anos de 2002 a 2019.

Os descritores utilizados foram: Idosos, Serviços de Assistência Domiciliar, Serviços de Saúde para Idosos, Atenção Primária à Saúde e os correspondentes em inglês. Após leitura dos artigos, a amostra constitui-se de 19 artigos, dentro dos critérios previamente definidos. Os critérios de inclusão foram artigos científicos que abordam a atenção domiciliar como assistência ao idoso. Os critérios de exclusão foram as produções que não se relacionavam ao serviço de assistência domiciliar.

\section{Resultados e Discussão}

\subsection{Perfil dos idosos que recebem Atendimento Domiciliar}

Dentre as literaturas explanadas, a maioria da população assistida em programas de atenção domiciliar são mulheres, fato que pode ser reflexo da maior expectativa de vida em mulheres em comparação com os homens. Ademais, os estudos relatam que a maior parte dos indivíduos que recebem atendimento domiciliar possuem como característica a presença de síndrome de fragilidade, baixa escolaridade e poder aquisitivo, associado a doenças crônicas (como hipertensão arterial sistêmica e diabetes mellitus) e que foram acometidos por quedas (Wachs, et al., 2016; Biscione, et al., 2013; Anderle, et al., 2013).

Em vista disso, é de grande importância uma intervenção multiprofissional voltada para a melhoria da qualidade de vida em específico desses usuários de forma integral, contínua e multidisciplinar, reinserindo o idoso em sua comunidade com estratégias de cuidado integral (Miranda, et al., 2016; Anderle, et al., 2013).

\subsection{A importância inicial da Assistência Domiciliar}

A atenção domiciliar (AD) surge com o objetivo de expandir o modelo de atenção historicamente realizado pelas práticas institucionalizadas, baseado no modelo hospitalocêntrico e biomédico, priorizando o valor da doença e não o indivíduo. A AD nasce em função das transformações no sistema de saúde e das mudanças sociais, e seu propósito é a inserção do profissional de saúde no domicílio, na convivência familiar do paciente e em sua cultura de vida, atuando na busca pela integralidade do idoso, na tentativa de ampliar sua autonomia e sua qualidade de vida (Medeiros, et al., 2017; Lacerda, et al., 2006).

A atenção domiciliar permite assistência ao usuário que não consegue ir ao atendimento na Unidade de Saúde da Família (USF) por limitações próprias do território do usuário (escassez de transporte público e difícil mobilidade urbana), principalmente os idosos que, geralmente, já convivem com problemas de locomoção. Ela permite também uma maior aproximação da equipe de profissionais com o idoso, além de serem bons ouvintes durante o cuidado (Schenker \& Costa, 2019). A AD pode ampliar a autonomia do idoso e de seus familiares e, se bem organizada, a integralidade e singularidade do cuidado pode ser assegurada por meio de medidas de prevenção, tratamento e educação em saúde no âmbito da Atenção Primária à Saúde (Medeiros, et al., 2017).

Biscione, et al., (2013) e Oliveira, et al., (2012) relatam que a frequência de visitas domiciliares impacta significativamente no aumento do tempo livre de internação em hospitais, diminuindo os efeitos deletérios das hospitalizações, bem como a redução da longa espera em um serviço de pronto atendimento, proporcionando também liberdade dentro do próprio espaço do paciente, com as regras e normas singulares da família.

Em relação aos custos hospitalares, o atendimento domiciliar promove o aumento da disponibilidade de leitos e assim, a redução de possíveis gastos com o enfermo que fica internado no hospital. Demonstrou-se que o programa "Melhor em casa" (MemC), associado ao atendimento domiciliar, reduziu os valores gastos com as internações em até 9,3\% relativo ao grupo de 
pessoas idosas atendidas no período de 2011 a 2013 (Brasil, 2012; Nishimura, et al., 2019). Desta forma, o resultado econômico com a redução de gastos relativo às internações, resulta em uma maior eficiência na gestão dos recursos orçamentários públicos, salvando também a saúde das contas públicas. Porém, ainda há necessidade de mais avaliações criteriosas e estudos acerca da relação custo-benefício da AD.

Desta forma, o atendimento domiciliar tem um grande papel de ampliar o vínculo das equipes de saúde para com a população, especialmente para o paciente, sua família e seu lar, tornando-se uma potencial ferramenta de cuidado complementar ou substitutiva à assistência hospitalar, mas que sua efetividade precisa ser melhor avaliada.

\subsection{Desafios da Assistência e Atenção Domiciliar}

Em determinado momento, o idoso pode se deparar na condição de necessidades de cuidados domiciliares, em decorrência de limitações físicas e psicológicas que podem vir a ocorrer por uma doença crônica ou acidente. E quem precisará assumir a responsabilidade do cuidado? A família, quando presente, junto com o apoio imprescindível do cuidador, formal ou informal. Dessa forma, é necessário discorrer inicialmente acerca dos desafios enfrentados pelos cuidadores, na tentativa de sanar tais obstáculos dentro desse serviço de assistência (Donati, et al., 2018).

Estudos mostraram as repercussões causadas no dia a dia de cuidadores de idosos e a suas realidades enfrentadas na assistência domiciliar. Demonstrou-se que a maioria dos cuidadores são mulheres, muitas vezes idosas, de baixa escolaridade, com multitarefas. Abandonar o próprio lazer e a liberdade em função da tarefa de cuidar, a falta de informação e orientação na execução dos procedimentos, insegurança no ato de cuidar do idoso, além do desgaste físico (cansaço físico, dores na coluna) e psicológico (estresse e preocupação) em uma rotina desgastante como cuidador são as principais repercussões negativas em seu cotidiano, sujeito a riscos ergonômicos e biológicos. Essa sobrecarga de tarefas desgastantes com repetitividade diária leva a outra problemática, que é a qualidade de vida do cuidador, com implicações que a tarefa de ser cuidador trás. É preciso orientar a forma correta de cuidar, ofertar suporte e reduzir a sobrecarga relacionada ao trabalho de cuidar (Floriani \& Schramm, 2004; Santos, et al., 2019; Guerra, et al., 2017).

Sérios problemas de recurso geram conflitos comprometendo a qualidade do serviço como a falta de suporte e apoio aos cuidadores, que podem ser informais ou formais. Fornecer suporte aos cuidadores é de extrema importância, em um lugar com condições mínimas de higiene e de segurança, assegurando a efetividade do cuidado (Padovani, et al., 2018).

Além disso, a decisão pelo atendimento no domicílio deve ser tomada com a garantia de que o ambiente tenha condições estruturais, e principalmente, a garantia de preparo da família, a qual terá um papel primordial no amparo ao idoso. Com a realidade social e econômica no país, na qual muitos brasileiros estão em situações de vulnerabilidade socioeconômica (em especial os idosos), a assistência domiciliar torna-se mais complexa, pois um domicílio sem o mínimo de estrutura física e segurança torna-se um obstáculo para o cuidador e para o idoso, principalmente quando seu envelhecimento implica um risco aumentado para o desenvolvimento de vulnerabilidades biológicas, socioeconômicas e psicossocial (Rodrigues \& Neri, 2012).

Percebe-se, portanto, que o atendimento domiciliar possui fragilidades relatadas na maioria das literaturas que envolvem o tema. Outros estudos mostram com menos frequência a situação de idosos que não contam com o apoio familiar, morando de forma solitária ou quando mora, o convívio familiar é debilitado. A violência contra idosos, além do abuso financeiro também é uma questão quando os maus tratos vêm do seu cuidador familiar. Há idosos que não se adaptam ao atendimento domiciliar pelo medo da violência e isso precisa ser debatido com respeito e segurança entre a equipe de saúde para um apontamento e enfrentamento de uma possível violência doméstica ou quaisquer situações adversas (Schenker, et al., 2019; Morais, et al., 2008). 


\section{Considerações Finais}

Esse estudo mostra, a partir da revisão de literatura, a necessidade de fortalecimento dessa modalidade terapêutica em vários âmbitos.

De modo geral, percebe-se que os estudos encontrados na pesquisa se preocupam com as fragilidades e desafios que a Assistência Domiciliar pode gerar. As repercussões sérias na saúde do cuidador, que geralmente é idoso, como desgaste físico e mental e carga horária extensa chamam a atenção e devem ser foco de intervenções da equipe de saúde, a fim de sanar os possíveis riscos ergonômicos e biológicos para com o cuidador.

Um dos principais objetivos da AD, segundo a portaria $\mathrm{n}^{\circ} 825$, de 25 de abril de 2016 é a otimização dos recursos financeiros e estruturais da Rede de Atenção à Saúde (RAS). Contudo, achados sobre a efetividade desse objetivo são escassos e baseados em um pequeno número de estudos, o que reforça a importância do gestor na operação de recursos nessa modalidade e a necessidade de novas pesquisas, especialmente para especificar os fatores eficazes no tratamento domiciliar.

É importante reforçar a necessidade de maior investimento para o sistema de saúde, para garantir as condições básicas de atendimento dentro de casa, além de garantir uma atenção integral ao idoso através da prevenção e educação em saúde nesse novo rearranjo de atendimento, onde o âmbito familiar, as culturas e tradições do indivíduo são tão importantes para a melhora da qualidade de vida quanto o tratamento e a cura. Foi demonstrado pelos autores a necessidade da saúde integral do idoso, além de ter sido discutidas maneiras de como fortalecer o cuidado físico, mental e social do usuário por meio da $\mathrm{AD}$, respeitando as diferenças e especificidades de cada indivíduo.

Conjectura-se, por fim, uma grande busca na reorganização do processo de trabalho pela equipe de saúde, na tentativa de ir além das práticas institucionalizadas, com reestruturação das práticas de saúde fora dos muros dos hospitais, a fim de garantir um cuidado humanizado centrado no paciente. Ademais, a construção de estudos observacionais que possam avaliar variáveis como qualidade de vida do idoso e dos cuidados, manejo de recursos econômicos e humanos, capacitação dos profissionais e cuidadores e percepção dos atores envolvidos na AD poderão auxiliar na reestruturação e implementação dos serviços oferecidos pela RAS.

\section{Referências}

Anderle, P., Souza, B. B., Julião, G. G., Millão, L. F., Santos, C. J., \& Monteiro, J. A. S. (2013). Perfil dos pacientes assistidos pela residência integrada em saúde: um olhar humanizado na assistência domiciliar. Aletheia, (41), 164-173.

Biscione, F. M., Szuster, D. A. C., Drumond, E. F., Ferreira, G. U. A., Turci, M. A., Lima Júnior, J. F., \& Bersan, S. A. L. (2013). Avaliação de efetividade da atenção domiciliar de uma cooperativa médica de Belo Horizonte, Minas Gerais, Brasil. Cadernos de Saúde Pública, 29(1), s73-s80.

Brasil. Ministério da Saúde. (2006). Agência Nacional de Vigilância Sanitária. RDC nº 11, de 26 de janeiro de 2006 . Dispõe sobre o regulamento técnico de funcionamento de serviços que prestam atenção domiciliar. Brasília, DF: Ministério da Saúde.

Brasil. Ministério da Saúde. (2012). Caderno de Atenção Domiciliar. Volume 1. Departamento de Atenção Básica, Secretaria de Atenção à Saúde, Ministério da Saúde.

Brasil. Ministério da Saúde. (2006). Departamento de Atenção Básica. Secretaria de Atenção à Saúde. Envelhecimento e saúde da pessoa idosa - Brasília DF: Ministério da Saúde.

Brasil. Ministério da Saúde. (2016). Portaria no 825, de 25 de abril de 2016. Redefine a Atenção Domiciliar no âmbito do Sistema Único de Saúde (SUS) e atualiza as equipes habilitadas [internet]. Brasília, DF: Ministério da Saúde.

Carvalho, V. L., \& Pereira, E. M. (2001). Crescendo na diversidade pelo cuidado domiciliar aos idosos: desafios e avanços. Revista Brasileira de Enfermagem,54 (1), 07-17.

Donati, L., Beuter, M., \& Schimith, M. D. (2018). Organização do Cuidado ao Idoso Dependente / Organization of Care to the Dependent Elderly. Textos \& Contextos (Porto Alegre), 17(1), 115 - 125.

Floriani, C. A. \& Schramm, F. R. (2004). Atendimento domiciliar ao idoso: problema ou solução? Cadernos de Saúde Pública, 20 (4), 986-994.

Guerra, H. S., Almeida, N. A. M., Souza, M. R. \& Minamisava, R. (2017). A sobrecarga do cuidador domiciliar. Rev. bras. promoç. Saúde. 30(2): 179-186

Guimarães, R. M. (2008). O mundo envelhece: é imperativo criar um pacto de solidariedade social. Ciência \& Saúde Coletiva, 13 (4), $1115-1117$. 
Research, Society and Development, v. 10, n. 3, e58810313775, 2021

(CC BY 4.0) | ISSN 2525-3409 | DOI: http://dx.doi.org/10.33448/rsd-v10i3.13775

Kalache, A. (2008). O mundo envelhece: é imperativo criar um pacto de solidariedade social. Ciência \& Saúde Coletiva, 13 (4), 1107-1111.

Lacerda, M. R., Giacomozzi, C. M., Oliniski, S. R., \& Truppel, T. C. (2006). Atenção à saúde no domicílio: modalidades que fundamentam sua prática. Saúde e Sociedade, 15(2), 88-95.

Medeiros, K. K. A. S., Pinto Júnior, E. P., Bousquat, A., \& Medina, M. G. (2017). O desafio da integralidade no cuidado ao idoso, no âmbito da Atenção Primária à Saúde. Saúde em Debate, 41 (spe), 288-295.

Miranda, G. M. D., Mendes, A. C. G. \& Silva, A. L. A. (2016). O envelhecimento populacional brasileiro: desafios e consequências sociais atuais e futuras. Revista Brasileira de Geriatria e Gerontologia, 19(3), 507-519.

Muñoz, S. I. S., Takayanagui, A. M. M., Santos, C. B. \& Sanchez-Sweatman, O. (2002). Revisão sistemática de literatura e metanálise: noções básicas sobre seu desenho, interpretação e aplicação na área da saúde. Simpósio Brasileiro de comunicação de Enfermagem. Escola de Enfermagem de Ribeirão Preto USP.

Moraes, C. L., Apratto Júnior, P. C., \& Reichenheim, M. E. (2008). Rompendo o silêncio e suas barreiras: um inquérito domiciliar sobre a violência doméstica contra idosos em área de abrangência do Programa Médico de Família de Niterói, Rio de Janeiro, Brasil. Cadernos de Saúde Pública, 24(10), 2289-2300.

Nishimura, F., Carrara, A. F., \& Freitas, C. E. (2019). Efeito do programa Melhor em Casa sobre os gastos hospitalares. Revista de Saúde Pública, 53, 104. Epub December 02, 2019.

Oliveira, S. G., Quintana, A. M., Budó, M. L. D., Kruse, M. H. L., \& Beuter, M. (2012). Internação domiciliar e internação hospitalar: semelhanças e diferenças no olhar do cuidador familiar. Texto \& Contexto - Enfermagem, 21(3), 591-599.

Padovani, C., Lopes, M. C. L., Higahashi, I. H., Pelloso, S. M., Paiano, M. \& Christophoro, R. (2018). Ser cuidador de pessoas com a Doença de Parkinson: situações vivenciadas. Revista Brasileira de Enfermagem, 71(6), 2628-2634.

Rodrigues, N. O. \& Neri, A. L. (2012). Vulnerabilidade social, individual e programática em idosos da comunidade: dados do estudo FIBRA, Campinas, SP, Brasil. Ciência \& Saúde Coletiva, 17(8), 2129-2139.

Santos W. P., Freitas F. B. D., Sousa V. A. G., Oliveira A. M. D., Santos J. M. M. P. \& Gouveia B. L. A. (2019). Sobrecarga de cuidadores idosos que cuidam de idosos dependentes. Rev Cuid.10(2): e607.

Schenker, M. \& Costa, D. H. (2019). Avanços e desafios da atenção à saúde da população idosa com doenças crônicas na Atenção Primária à Saúde. Ciência \& Saúde Coletiva, 24(4), 1369-1380.

Silva, K. L., Sena, R. R., Seixas, C. T., Feuerwerker, L. C. M., \& Merhy, E. E. (2010). Atenção domiciliar como mudança do modelo tecnoassistencial. Revista de Saúde Pública, 44(1), 166-176.

Wachs, L. S., Nunes, B. P., Soares, M. U., Facchini, L. A. \& Thumé, E. (2016). Prevalência da assistência domiciliar prestada à população idosa brasileira e fatores associados. Cadernos de Saúde Pública, 32(3), e00048515. 